\title{
A case of cornual pregnancy at ten gestational age accompanied by heterotopic pregnancy
}

\author{
Jisun Yun, Jong-wook Seo, In Ok Lee, Jae Eun Chung*
}

Department of Obstetrics and Gynecology, National Health Insurance Service Ilsan Hospital, Goyang, Republic of Korea

Received: 29 October 2020

Accepted: 09 December 2020

\author{
*Correspondence: \\ Dr. Jae Eun Chung, \\ E-mail: obgy0278@nhimc.or.kr
}

Copyright: (c) the author(s), publisher and licensee Medip Academy. This is an open-access article distributed under the terms of the Creative Commons Attribution Non-Commercial License, which permits unrestricted non-commercial use, distribution, and reproduction in any medium, provided the original work is properly cited.

\begin{abstract}
Objective of the study was to report a case of cornual pregnancy at 10 weeks gestational age, accompanied by heterotopic pregnancy. A 41-year-old woman was transferred to the emergency department of the national health insurance service Ilsan hospital at amenorrhea 10 weeks 5 days after being diagnosed of cornual pregnancy from a primary hospital. The patient was nulliparous with a history of 1 induced and 2 missed abortions. Other than a previous myomectomy, her medico-surgical history was not significant. She was initially diagnosed with blight ovum at amenorrhea 8 weeks 4 days, and dilatation and curettage were performed at the primary hospital of which she was referred from. At amenorrhea 10 weeks 5 days, another gestational sac was noted at the left cornu of the uterus by ultrasonography. The tentative diagnosis was cornual pregnancy accompanied by heterotopic pregnancy. Serum $\beta$ human chorionic gonadotrophin (hCG) at the time of arrival was $84573.8 \mathrm{mIU} / \mathrm{ml}$. Trans-abdominal ultrasonography showed a mass protruding from the left cornu of the uterine cavity, containing a gestational sac (mean sac diameter $39.0 \mathrm{~mm}, 10$ weeks size) and an embryo of cranio-caudal length of $38.2 \mathrm{~mm}$ (10 weeks 5 days size). There was no sign of intraperitoneal hemorrhage. Emergency operation was planned under the diagnosis of cornual pregnancy. Entering the abdominal cavity, a $5 \times 5 \mathrm{~cm}$ sized conceptual mass surrounded by thin and fragile myometrium was noted on left uterine cornu. Open wedge resection of the left cornual pregnancy with left salpingectomy was performed. The resected mass was $4.0 \times 3.5 \mathrm{~cm}^{2}$ in size. An embryo with $45 \mathrm{~mm}$ size was confirmed inside the mass.
\end{abstract}

Keywords: Cornual pregnancy, Heterotopic pregnancy, Gestational age 10 weeks, Myomectomy

\section{INTRODUCTION}

Ectopic pregnancy is defined as a condition where the fertilized egg is implanted in a place other than the endometrium of the uterus. It is diagnosed in early pregnancy and requires an emergency operation due to the highly associated maternal mortality and morbidity. Ectopic pregnancy occurs in the fallopian tubes in about $95 \%$ of the cases, and the rest of the cases occur in the uterine cornu, ovaries, abdominal cavity, and the uterine cervix.

Cornual pregnancy refers to the implantation of the fertilized egg in the uterine cornu, which occurs in a much rarer setting. ${ }^{1-3}$ Cornual pregnancy results in a higher maternal mortality rate than ectopic pregnancy in general. Due to the flexibility of the uterine muscles and abundant vascular distribution surrounding the implantation site, pregnancy is more likely to be delayed by 8 to 16 weeks compared to ectopic pregnancy in other areas. Cornual pregnancy eventually ruptures and causes massive bleeding. Early diagnosis before rupture is very important, however, it is very difficult to differentiate cornual pregnancy from intrauterine pregnancy in the early stages of pregnancy. Most uterine cornual pregnancies end up in emergency surgery due to massive intraperitoneal bleeding after its rupture.

Heterotopic pregnancy is defined as the coexistence of an ectopic and intrauterine pregnancy. The incidence of 
heterotopic pregnancy in naturally conceived pregnancy is relatively rare, but up to $1 \%$ of patients who undertake in vitro fertilization and embryo transfer may end up in heterotopic pregnancy. ${ }^{3}$

We present here a case of heterotrophic pregnancy where the cornual pregnancy that was not noted at the time of the pregnancy termination due to a blighted ovum. Without experiencing a massive hemorrhage due to the cornual pregnancy rupture, the case was successfully treated.

The presence of an intrauterine pregnancy does not rule out the presence of a coexisting ectopic pregnancy. Clinicians should be aware of the possibility of a heterotopic pregnancy and always be vigilant in checking the uterus and appendages even at the presence of intrauterine pregnancy.

\section{CASE REPORT}

A 41-years-old woman was transferred to the emergency department of national health insurance service Ilsan hospital at amenorrhea 10 weeks 5 days after being diagnosed of cornual pregnancy at a primary hospital on July 2020. The patient was nulliparous with 1 induced and 2 missed abortions. She had a history of taking a laparoscopic myomectomy 1 year ago. Her menstrual periods were regular (28 days), and the last menstruation date was April 29, 2020. She was diagnosed with an intrauterine pregnancy in her previous hospital but it ended up being a blighted ovum at amenorrhea 8 weeks 4 day. Dilatation and curettage of the blighted ovum was done without any complication, however, the symptoms of hyperemesis persisted for 2 weeks. On ultrasound examination, other gestational sac of 10 weeks 5 days size was noted in the left uterine cornu. She was diagnosed with cornual pregnancy, accompanied by heterotopic pregnancy, and was transferred to the emergency department.

On her arrival, the patient did not complain of typical symptoms such as abdominal pain or vaginal bleeding. Vital signs were stable; the blood pressure was 136/87 $\mathrm{mmHg}$, the pulse rate was 80 beats/min, and the axillary body temperature was $37.3^{\circ} \mathrm{C}$. On physical examination, she had left lower quadrant direct tenderness without rebound tenderness. Transabdominal and transvaginal ultrasonography showed a mass protruding from the left cornu of the uterine cavity, containing a gestational sac (mean sac diameter $39.0 \mathrm{~mm}, 10$ weeks size) and an embryo of cranio-caudal length of $38.2 \mathrm{~mm}$ (10 weeks 5 days size) (Figure 1).

Laboratory evaluation revealed haemoglobin $13.9 \mathrm{~g} / \mathrm{dl}$, haematocrit $40.1 \%$, white cell blood count $10990 / \mu 1$, and normal kidney and liver function test. Serum $\beta$ hCG level was $84573.8 \mathrm{mIU} / \mathrm{ml}$. There was no evidence of intraperitoneal haemorrhage or ruptured cornual pregnancy on clinical, laboratory and ultrasonographic finding. An emergency laparotomy was planned.

Entering the abdominal cavity, the diagnosis of left cornual pregnancy was confirmed. A $5 \times 5 \mathrm{~cm}^{2}$ sized protruding mass surrounded by thin fragile myometrium was noted on left uterine cornu extending to the left fallopian tube. Wedge resection of left cornual pregnancy and left salpingectomy was performed. Drained blood amount was minimal. The uterus, right ovary and fallopian tube, left ovary were normal. The resected mass was $4.0 \times 3.5 \mathrm{~cm}^{2}$ size. An embryo of $45 \mathrm{~mm}$ size was confirmed inside the mass. (Figure 2). Haemoglobin level at first postoperative day was $12.3 \mathrm{~g} / \mathrm{dl}$, and serum $\beta \mathrm{hCG}$ level was $35746.0 \mathrm{mIU} / \mathrm{ml}$. Patient recovered without any complication after the laparotomy, and was discharged on the third postoperative day.

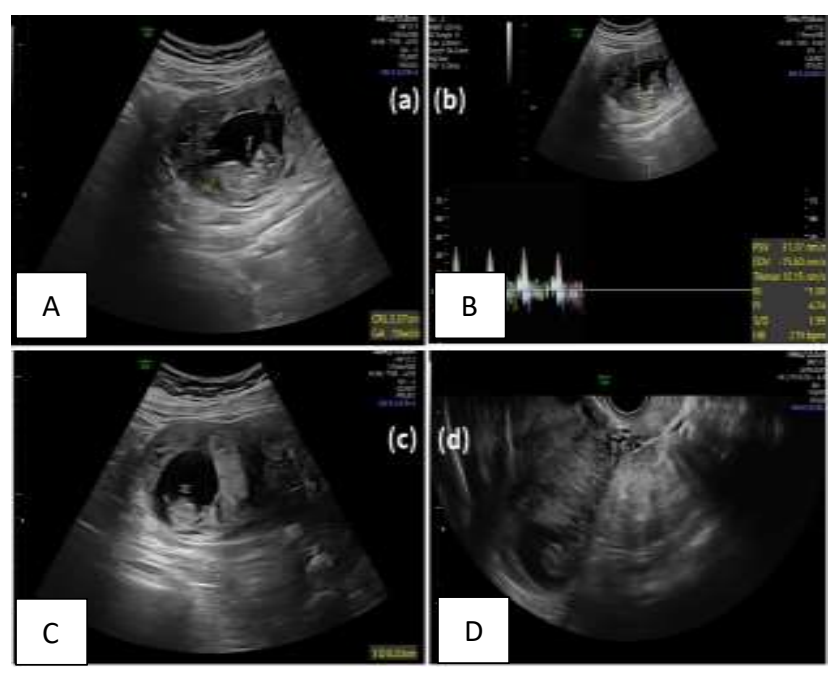

Figure 1: Trans-abdominal ultrasonographic scan, (A) Crown-Rump length of fetus was $3.37 \mathrm{~cm}$ (10 weeks 2 days size), (B) the fetal heart beat 219 bpm was checked, (C) thickness of myometrium was $2.3 \mathrm{~mm}$, (D) gestational sac was located on left uterine cornu.
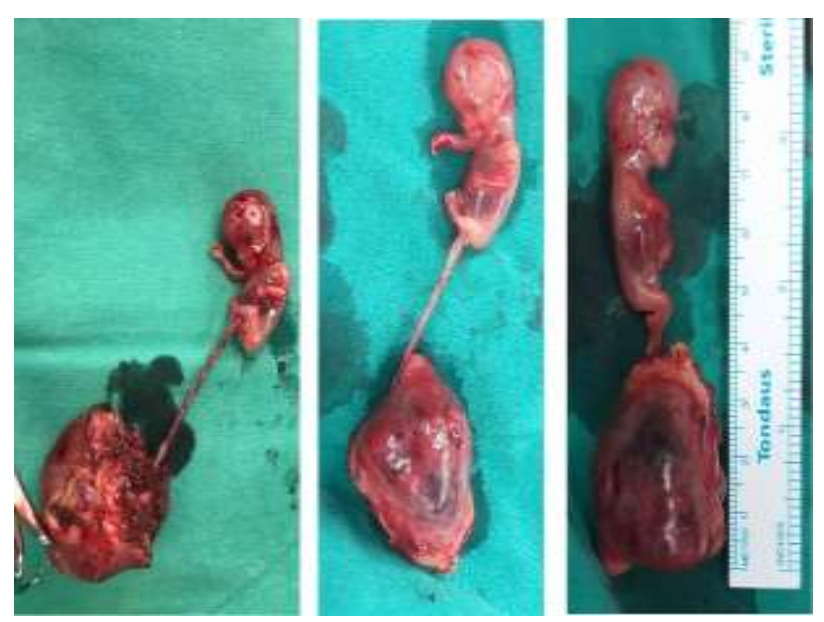

Figure 2: The resected mass was $4.0 \times 3.5 \mathrm{~cm}^{2}$ size. An embryo with $45 \mathrm{~mm}$ size was confirmed inside the mass. 


\section{DISCUSSION}

Uterine cornual pregnancy is a rare form of ectopic pregnancy in which a fertilized egg is implanted at the interstitial portion of the fallopian tube. It occurs rather rarely: only 4 cornual pregnancies were reported in 21 years of clinical experience at a single institution, covering 654 ectopic pregnancies. $^{1}$ The incidence remained low in other studies as well, varying from 0.5 to $0.7 \% .^{2}$ However, the incidence of ectopic pregnancy is on the rise especially in patients with a history of in vitro fertilization and embryo transfer procedures. Heterotopic cornual pregnancy is even more rare implicating a diagnostic and therapeutic challenge. ${ }^{4}$

The uterine cornu is the area with dense vascular tissue where the ovarian artery and the uterine artery form an anastomosis. In this area, the fallopian tube is surrounded by the uterine muscle, and has transitions to the uterus. When implantation is made in this area, the gestational sac is protected by the muscle layer of the uterus, and the pregnancy lasts longer than other tubal pregnancies. ${ }^{5,6}$ However, the chorionic villi invade the blood vessels in the uterine cornu, causing massive bleeding, which is associated with maternal mortality and morbidity. Rupture of cornual pregnancy mostly occur between 8and 16-weeks gestational age. A case of cornual pregnancy that did not rupture until 32 weeks into the pregnancy has been reported. ${ }^{7}$ Once ruptured, due to fatal bleeding, the reported mortality rate $45-65 \% .{ }^{8}$ But in recent years, maternal mortality has been decreasing with early diagnosis, appropriate blood transfusion, and immediate surgical treatment.

Cornual pregnancy can be diagnosed when one of the following three conditions is satisfied; clinical features with asymmetrical uterine growth with pain-associated miscarriage or vaginal delivery, the uterus expanding to lateral part and directly pushing the round ligament outward and the remnant placenta in the uterine cornus. ${ }^{2}$ The clinical symptoms of an un-ruptured cornual pregnancy are difficult to distinguish from any other ectopic pregnancy. Common symptoms include amenorrhea, vaginal bleeding, lower abdominal pain, or tenderness in the pelvis. There is no shock sign before rupture, but once ruptured, bleeding can be very severe and can be fatal, so the frequency at which shock can occur is 2-5 times higher than that of other tubal pregnancy.

Heterotopic pregnancy is very rare in spontaneous pregnancies and is known to be 1 case out of 700030000. ${ }^{9-11}$ But, as in vitro fertilization has been commercialized, the incidence is increasing to about $1 \%{ }^{4}$

Early diagnosis before rupture is very important for cornual pregnancy, but it is very difficult to differentiate between normal intrauterine pregnancy and cornual pregnancy. Ultrasonographic scan, laparoscopy, and culdocentesis are typical diagnostic techniques, and ultrasound is useful for determining the location of the gestational sac in the uterine cavity. The findings that can be diagnosed with ultrasound in uterine cornus are as follows; first, there was no gestational sac in the uterine cavity, second, the distance between the chorionic sac and the outer side of the uterine cavity is more than $1 \mathrm{~cm}$ apart, and third, a thin uterine muscle layer must surround the chorionic sac. ${ }^{6-12}$ In intrauterine pregnancy, the uterine muscle completely encloses the gestational sac, but in cornual pregnancy, the uterine muscle covers the gestational sac with a thin rim. On ultrasound, when the gestational sac is located in the uterine cornus, or is at the lateral part of uterine fundus, and clinically suspected intraperitoneal bleeding, a diagnostic laparoscopy should be performed immediately.

As with other ectopic pregnancies, cornual pregnancy seems to be more prevalent in cases of uterine malformations with fibroids, or Mullerian anomalies. As the past history of miscarriage is also frequently reported, the spatial problem of uterus seems to be the cause due to difficulty of proper implantation. ${ }^{13}$

Treatment depends on the patient's general condition, the likelihood of future pregnancy, the duration of pregnancy and the degree of uterine muscle involvement. If the foetus is alive and the gestational sac is completely covered by the uterine muscle, no special active treatment is required. ${ }^{2}$ On the other hand, if the uterine muscle is discoloured and bleeding, it will be ruptured soon. Therefore, it is necessary to perform wedge resection or partial uterine cornual resection. ${ }^{14,15}$ In women under 35 years of age, with early gestational age, (less than 8 weeks), if they want to become pregnant later, cornuotomy and wedge resection may be treatment options. ${ }^{16}$ Hysterectomy is performed at the advanced gestational age, or with severe uterine muscle involvement. In the case of cornuotomy and wedge resection, caesarean section is the most commonly accepted method of delivery in subsequent pregnancy because of the risk of rupture during delivery. ${ }^{17}$ If the patient wants to become pregnant and surgery is contraindicated, medications such as methotrexate may be tried. ${ }^{18-21}$

\section{CONCLUSION}

In conclusion, cornual pregnancy is rare, and it is very difficult to diagnose early before rupture due to its anatomical specificity. However, due to the risk of severe bleeding, early diagnosis of cornual pregnancy must be done. The authors experienced a case of uterine cornual pregnancy accompanied by heterotopic pregnancy in a woman who had previously undergone myomectomy. Since it was treated successfully, we report it with a brief review of the literature.

Funding: No funding sources Conflict of interest: None declared Ethical approval: Not required 


\section{REFERENCES}

1. Breen JL. A 21-year survey of 654 ectopic pregnancies. Am J Obstet Gynecol. 1970;106(7):1004-19.

2. Jansen RP, Elliott PM. Angular intrauterine pregnancy. Obstetrics Gynecol. 1981;58(2):167-75.

3. Murray $\mathrm{H}$, Baakdah $\mathrm{H}$, Bardell $\mathrm{T}$, Tulandi $\mathrm{T}$. Diagnosis and treatment of ectopic pregnancy. Canadian Medic Assoc J. 2005;173(8):905-12.

4. Divry V, Hadj S, Bordes A, Genod A, Salle B. Case of progressive intrauterine twin pregnancy after surgical treatment of cornual pregnancy. Fertil Steril. 2007;87(1):190:e1-3.

5. Coady D, Snyder J, Goldstein S, Subramanyan B. Ultrasound diagnosis of interstitial pregnancy. NY State J Med. 1985;85(11):655-6.

6. Sherer DM, Allen T, Singh GS, Woods Jr JR. Transvaginal sonographic diagnosis of an unruptured interstitial pregnancy. J Clin Ultrasound. 1990;18(7):582-5.

7. Song MH, Seong YM, Kim KJ. A case of cornual pregnancy found in 32 gestational weeks of pregnancy. Obstet Gynecol Sci. 1995;38(1):111-21.

8. Felmus LB, Pedowitz P. Interstitial pregnancy: a survey of 45 cases. Am J Obstet Gynecol. 1953;66(6):1271-9.

9. Dor J, Seidman DS, Levran D, Ben-Rafael Z, BenShlomo I, Mashiach S. The incidence of combined intrauterine and extrauterine pregnancy after in vitro fertilization and embryo transfer. Fertility sterility. 1991;55(4):833-4.

10. Dumesic DA, Damario MA, Session DR, editors. Interstitial heterotopic pregnancy in a woman conceiving by in vitro fertilization after bilateral salpingectomy. Mayo Clin Proceedings. 2001;76(1):90-2.

11. Pan H-S, Chuang J, Chiu S-F, Hsieh B-C, Lin Y-H, Tsai Y-L, et al. Heterotopic triplet pregnancy: report of a case with bilateral tubal pregnancy and an intrauterine pregnancy: Case report. Human Reproduction. 2002;17(5):1363-6.
12. Stiller RJ, de Regt RH. Prenatal diagnosis of angular pregnancy. J clinical ultrasound. 1991;19(6):374-6.

13. Deckers E, Stamm C, Naake V, Dunn T, McFee J. Hysterotomy for retained placenta in a term angular pregnancy. A case report. J Reprod med. 2000;45(2):153-5.

14. Sasso R. Laparoscopic diagnosis and treatment of cornual pregnancy. A case report. J Reprod Med. 1995;40(1):68-70.

15. Tulandi T, Vilos G, Gomel V. Laparoscopic treatment of interstitial pregnancy. Obstet Gynecol. 1995;85(3):465-7.

16. Lau S, Tulandi T. Conservative medical and surgical management of interstitial ectopic pregnancy. Fertil Steril. 1999;72(2):207-15.

17. Timor-Tritsch IE, Monteagudo A, Matera C, Veit CR. Sonographic evolution of cornual pregnancies treated without surgery. Obstet Gynecol. 1992;79(6):1044-9.

18. Barnhart K, Spandorfer S, Coutifaris C. Medical treatment of interstitial pregnancy. A report of three unsuccessful cases. J Reprod Med. 1997;42(8):5214.

19. Benifla J-L, Fernandez H, Sebban E, Darai E, Frydman R, Madelenat P. Alternative to surgery of treatment of unruptured interstitial pregnancy: 15 cases of medical treatment. Eur J Obstet Gynecol Reprod Biol. 1996;70(2):151-6.

20. Brandes MC, Youngs DD, Goldstein DP, Parmley TH. Treatment of cornual pregnancy with methotrexate: case report. Am J Obstet Gynecol. 1986;155(3):655-7.

21. Chandra P, Koenigsberg M, Romney SL, Koren Z, Schulman H. Unruptured interstitial pregnancy. Diagnosis and treatment. Obstet Gynecol. 1978;52(5):612-5.

Cite this article as: Yun J, Seo JW, Lee IO, Chung JE. A case of cornual pregnancy at ten gestational age accompanied by heterotopic pregnancy. Int $\mathrm{J}$ Reprod Contracept Obstet Gynecol 2021;10:343-6. 